\title{
Synthesis, Characterization and Antioxidant Activity of Carvacrol Based Sulfonates
}

Suresh D Bagul, Jamatsing D Rajput, Manohar M Patil and Ratnamala S Bendre*

Department of Pesticides and Agrochemicals, School of Chemical Sciences, North Maharashtra University, Jalgaon 425001, Maharashtra, India

\begin{abstract}
In the present investigation, we report eight novel benzenesulfonate derivatives of carvacrol prepared by using sulfonyl chlorides and carvacrol. Their structures were investigated on the basis of modern sophisticated analytical techniques such as ${ }^{1} \mathrm{H}$ and ${ }^{13} \mathrm{C}$ NMR, LC-MS and FT-IR spectroscopy. The synthesized derivatives are screened for their antioxidant test by DPPH radical scavenger assay. Among the tested compounds, $\mathbf{6} \mathbf{g}$ and $\mathbf{6} \mathbf{h}$ have emerged as better antioxidants.
\end{abstract}

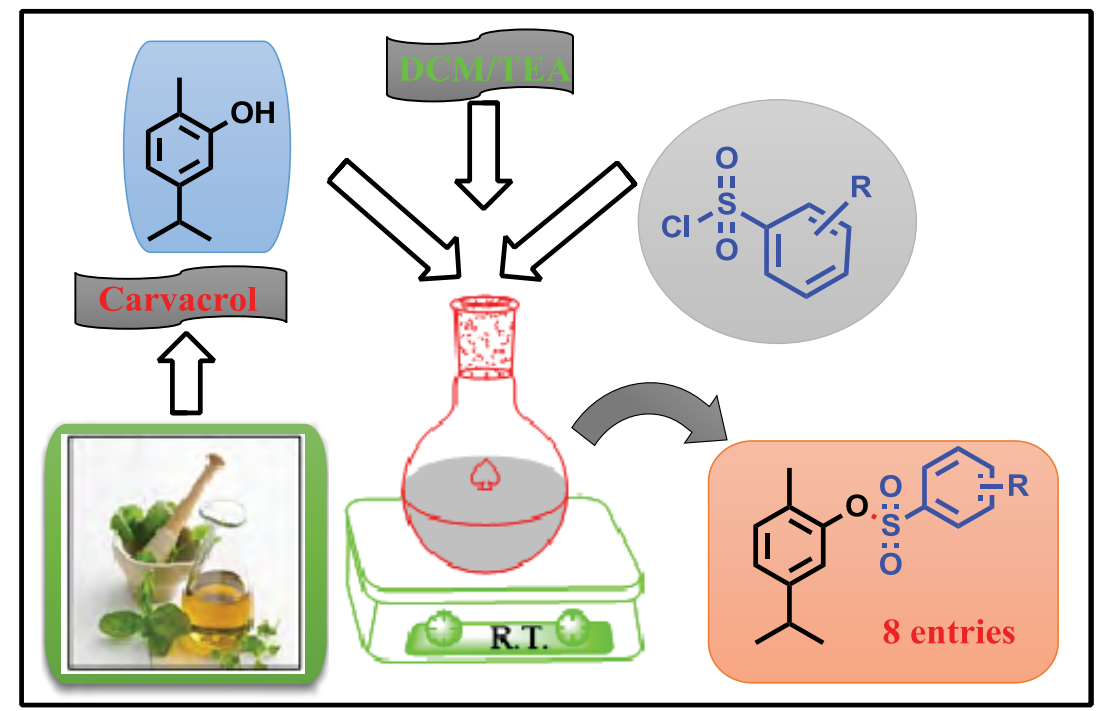

Keywords: Carvacrol; Sulfonyl chloride; Benzenesulfonate; DPPH; Antioxidant activity

\section{Introduction}

Nowadays antioxidants have motivated researchers' interest in both medicinal plants and synthetic organic compounds [1]. The implication of free radicals and reactive oxygen species (ROS) has been found to be in the pathogenicity of numerous diseases, including various chronic diseases [2,3]. Antioxidants are recently invented as the drug candidates to counter these diverse diseases, such as carcinogenesis, inflammation, and aging in aerobic organisms [4]. The design of small molecular agents to conflict cellular oxidative stress has become an important therapeutic objective, towards comprehensive damage to cellular macromolecules caused by reactive oxygen species (ROS) [5]. The extensive action of synthetic antioxidants is being ruled out owing to their toxicity and unwanted side effects and there is a growing interest in the use of the natural product as antioxidants and their derivatives for the treatment of oxidative stress-related diseases [6].

The antioxidant activity of phenols has been found depending on the electronic and steric effects of the ring, substituents and the strength of hydrogen-bonding interactions between phenol and solvent $[7,8]$. Many essential oils exhibit antioxidant and antimicrobial activities [9]. Phenols, such as thymol, carvacrol, eugenol and monocyclic hydrocarbons belong to the most active natural antioxidants found in the essential oils. However, the efficiency of these compounds in treatment is limited due to their poor water solubility and the requirement of high concentrations to reach a therapeutic effect [10,11]. Carvacrol, 5-isopropyl-3-methylphenol (Figure 1 (1)) is a major constituent of organo oil [12-15]. It is a phenolic monoterpenoid that exhibits several interesting biological activities [16-18]. It has antiinflammatory [19], antibiotic [20], antifungal [21], antioxidant [22], antiviral [23], insecticidal [24], cardioprotective [25] and antidiabetics [26] properties. Josip et al. synthesized 4-(hydroxymethyl)-5-isopropyl2-methylphenol (Figure 1 (2)) and 4,4'-methylenebis(5-isopropyl-2methyl)phenol (Figure 1 (3)) and studied their antioxidant activity [27].

In this work, we have synthesized carvacrol sulfonate derivatives and characterized them by sophisticated analytical techniques. Antioxidant properties of these compounds have been investigated by in vitro systems through the interaction of 2,2-diphenyl-1-picrylhydrazyl

*Corresponding author: Ratnamala $S$ Bendre, Department of Pesticides and Agrochemicals, School of Chemical Sciences, North Maharashtra University, Jalgaon-425 001, Maharashtra, India, Tel: 8659264596; E-mail: bendrers@rediffmail.com

Received October 05,2017; Accepted October 13,2017; Published October 18,2017

Citation: Bagul SD, Rajput JD, Patil MM, Bendre RS (2017) Synthesis, Characterization and Antioxidant Activity of Carvacrol Based Sulfonates. Med Chem 7: 294-298. doi: 10.4172/2161-0444.1000470

Copyright: @ 2017 Bagul SD, et al. This is an open-access article distributed unde the terms of the Creative Commons Attribution License, which permits unrestricted use, distribution, and reproduction in any medium, provided the original author and source are credited. 


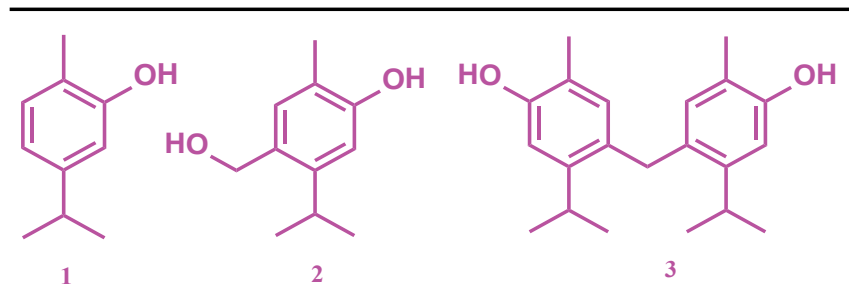

Figure 1: Structures of Carvacrol, 4-hydroxymethyl carvacrol and Dimer of carvacrol.

(DPPH) and scavenging of superoxide radical.

\section{Experimental}

\section{Chemicals and instruments}

Melting points of all the synthesized compounds were determined by the open capillary method. The confirmation of synthesized compounds was checked by thin layer chromatography on $200 \mu \mathrm{m}$ thick aluminum sheets having silica gel $60 \mathrm{~F}_{254}$ as an adsorbent by developing the TLC plate using hexane: ethyl acetate (4:1) solvent system. Spots were visualized under UV-light. ${ }^{1} \mathrm{H}$ and ${ }^{13} \mathrm{C}$ NMR spectra were scanned at Bruker AC-400 $\mathrm{MHz}$ spectrometer FT NMR in $\mathrm{CDCl}_{3}$ using TMS as an internal standard. The chemical shift values are on $\delta$ scale. The mass spectra were recorded on LC-MS spectrometer. All chemicals and solvents were locally purchased from Sigma-Aldrich and S. D. Fine chemicals and used without further purification.

\section{General procedure for synthesis of carvacrol benzenesulfonate}

Carvacrol (4) (1 mL, 0.006 moles) in dichloromethane $(5 \mathrm{~mL})$ was charged to the round bottom flask, then triethylamine $(1 \mathrm{~mL}, 0.009$ moles) was added drop-wise to it. Substituted benzene sulfonyl chloride (0.006 moles) (5) was added to it with constant stirring for 5-6 hours at room temperature. Progress of the reaction was monitored by TLC for every $15^{\text {th }}$ minute. Upon confirmation of completion of reaction, $10 \% \mathrm{NaHCO}_{3}$ solution was added and the reaction mixture was further stirred for 30 minutes. The reaction mixture was extracted by using separating funnel and the organic layer evaporated at room temperature $[28,29]$. The remaining solid/liquid was collected and recrystallized with suitable solvent. Melting / Boiling point and practical yield were recorded. A detailed description of the spectral data for compounds is provided in the Supporting Information.

5-isopropyl-2-methylphenyl 2-fluorobenzenesulfonate (6a): ${ }^{1} \mathrm{H}$ NMR $\left(100 \mathrm{MHz}, \mathrm{CDCl}_{3}\right) \delta 1.09-1.11(6 \mathrm{H}, \mathrm{d}, \mathrm{J}=6.87 \mathrm{~Hz}), 2.22(3 \mathrm{H}$, s), 2.73-2.88 $(1 \mathrm{H}, \mathrm{m}), 6.76(1 \mathrm{H}, \mathrm{s}), 7.25(1 \mathrm{H}, \mathrm{d}, \mathrm{J}=7.9 \mathrm{~Hz}), 7.12(1 \mathrm{H}$, d, J=7.9 Hz), $7.26(1 \mathrm{H}, \mathrm{t}, \mathrm{J}=7.9 \mathrm{~Hz}), 7.32(1 \mathrm{H}, \mathrm{d}, \mathrm{J}=7.9 \mathrm{~Hz}), 7.66(1 \mathrm{H}$, t, J=7.9 Hz), $7.85(1 \mathrm{H}, \mathrm{d}, \mathrm{J}=7.9 \mathrm{~Hz}),{ }^{13} \mathrm{C}$ NMR $\left(100 \mathrm{MHz}, \mathrm{CDCl}_{3}\right) \delta$ $16.81,23.11,33.38,17.01,117.43-117.64,119.74,124.44-125.43$, 128.66, 131.38-131.52, 136.63-136.72, 148.01-148.18, 158.25, 161.14 FT-IR (KBr, cm $\left.{ }^{-1}\right) 3090$ (Aromatic C-H Stretch), 1575 (Aromatic C=C Bending), 1489 (C-F Stretching), 1386 and 1266 ( $\mathrm{SO}_{2}$ Stretching), 1067 (C-O Stretching), 815 and 775 (Aromatic C-H Bending), LC-MS m/z calculated $\mathrm{C}_{16} \mathrm{H}_{17} \mathrm{FO}_{3} \mathrm{~S}: 308.37$ found: $[\mathrm{M}+\mathrm{Na}]^{+} 331.26$.

5-isopropyl-2-methylphenyl 4-fluorobenzenesulfonate (6b): ${ }^{1} \mathrm{H}$ NMR $\left(400 \mathrm{MHz}, \mathrm{CDCl}_{3}\right) \delta 1.13(6 \mathrm{H}, \mathrm{d}, \mathrm{J}=6.87 \mathrm{~Hz}), 2.02(3 \mathrm{H}, \mathrm{s})$, 2.75-2.81 $(1 \mathrm{H}, \mathrm{m}), 6.64(1 \mathrm{H}, \mathrm{s}), 6.99(1 \mathrm{H}, \mathrm{d}, \mathrm{J}=7.9 \mathrm{~Hz}), 7.16(1 \mathrm{H}, \mathrm{d}$, $\mathrm{J}=7.9 \mathrm{~Hz}), 7.19(2 \mathrm{H}, \mathrm{d}, \mathrm{J}=7.9 \mathrm{~Hz}), 7.81(2 \mathrm{H}, \mathrm{d}, \mathrm{J}=7.9 \mathrm{~Hz}),{ }^{13} \mathrm{C} \mathrm{NMR}$ $\left(100 \mathrm{MHz}, \mathrm{CDCl}_{3}\right) \delta 15.93,23.15,33.41,76.06-77.49,116.43-116.65$, $128.11,131.35-132.19,148.16-148.21,164.15,167.34$, FT-IR $(\mathrm{KBr}$, $\mathrm{cm}^{-1}$ ) 3061 and 2964 (Aromatic C-H Stretch), 1589 (Aromatic C=C Bending), 1499 (C-F Stretching), 1319 and 1192 (-SO Stretching), 1079 (C-O Stretching), 796 (Aromatic C-H Bending), LC-MS m/z calculated
$\mathrm{C}_{16} \mathrm{H}_{17} \mathrm{FO}_{3} \mathrm{~S}: 308.37$ found: $[\mathrm{M}+\mathrm{Na}]^{+} 331.27$.

5-isopropyl-2-methylphenyl 2-chlorobenzenesulfonate (6c): ${ }^{1} \mathrm{H}$ NMR $\left(400 \mathrm{MHz}, \mathrm{CDCl}_{3}\right) \delta 1.09-1.12(6 \mathrm{H}, \mathrm{d}, \mathrm{J}=6.87 \mathrm{~Hz}), 2.22(3 \mathrm{H}, \mathrm{s})$, 2.71-2.81 $(1 \mathrm{H}, \mathrm{m}), 6.99(1 \mathrm{H}, \mathrm{s}), 7.28(1 \mathrm{H}, \mathrm{d}, \mathrm{J}=7.9 \mathrm{~Hz}), 7.66(1 \mathrm{H}, \mathrm{d}$, $\mathrm{J}=7.9 \mathrm{~Hz}), 7.68(1 \mathrm{H}, \mathrm{t}, \mathrm{J}=7.9 \mathrm{~Hz}), 7.71(1 \mathrm{H}, \mathrm{d}, \mathrm{J}=7.9 \mathrm{~Hz}), 7.82(1 \mathrm{H}, \mathrm{t}$, $\mathrm{J}=7.9 \mathrm{~Hz}), 7.86(1 \mathrm{H}, \mathrm{d}, \mathrm{J}=7.9 \mathrm{~Hz}),{ }^{13} \mathrm{C} \mathrm{NMR}\left(100 \mathrm{MHz}, \mathrm{CDCl}_{3}\right) \delta 15.42$, 22.88-23.12, 38.83-40.08, 64.50, 109.37, 123.33, 128.00-129.55, 131.47, 132.98, 142.42, 153.71, FT-IR $\left(\mathrm{KBr}, \mathrm{cm}^{-1}\right) 3066$ and 2958 (Aromatic C-H Stretch), 1535 (Aromatic C=C Bending), 1425 (C-F Stretching), 1282 and 1190 (-SO Stretching), 1008 (C-O Stretching), 867 (Aromatic $\mathrm{C}-\mathrm{H}$ Bending), LC-MS m/z calculated $\mathrm{C}_{16} \mathrm{H}_{17} \mathrm{ClO}_{3} \mathrm{~S}$ : 324.82 found: $[\mathrm{M}+\mathrm{H}]^{+} 325.24$.

5-isopropyl-2-methylphenyl 4-chlorobenzenesulfonate (6d): ${ }^{1} \mathrm{H}$ NMR $\left(400 \mathrm{MHz}, \mathrm{CDCl}_{3}\right) \delta 1.11-1.14(6 \mathrm{H}, \mathrm{d}, \mathrm{J}=6.87 \mathrm{~Hz}), 2.01(3 \mathrm{H}$, s), 2.15-2.82 (1H, m), $6.73(1 \mathrm{H}, \mathrm{s}), 7.02(1 \mathrm{H}, \mathrm{d}, \mathrm{J}=7.9 \mathrm{~Hz}), 7.2(1 \mathrm{H}, \mathrm{d}$, $\mathrm{J}=7.9 \mathrm{~Hz}), 7.82(2 \mathrm{H}, \mathrm{d}, \mathrm{J}=7.9 \mathrm{~Hz}), 9.02(2 \mathrm{H}, \mathrm{d}, \mathrm{J}=7.9 \mathrm{~Hz}){ }^{13} \mathrm{C}$ NMR $(100$ $\left.\mathrm{MHz}, \mathrm{CDCl}_{3}\right) \delta 15.95,23.15,33.41,76.11,77.46,116.41-116.63,120.88$, $125.48,128.68,131.34-132.19,148.14-148.21,164.14$, FT-IR $(\mathrm{KBr}$ $\left.\mathrm{cm}^{-1}\right) 3273$ and 2972 (Aromatic C-H Stretch), 1517 (Aromatic C=C Bending), 1427 (C-F Stretching), 1394and 1276 (-SO Stretching), 1020 (C-O Stretching), 879 (Aromatic C-H Bending), LC-MS m/z calculated $\mathrm{C}_{16} \mathrm{H}_{17} \mathrm{ClO}_{3} \mathrm{~S}: 324.82$ found: $[\mathrm{M}+\mathrm{H}]^{+} 325.03$.

5-isopropyl-2-methylphenyl 2-bromobenzenesulfonate (6e): ${ }^{1} \mathrm{H}$ NMR $\left(400 \mathrm{MHz} \mathrm{CDCl}_{3}\right) \delta 1.12-1.14(6 \mathrm{H}, \mathrm{d}, \mathrm{J}=6.87 \mathrm{~Hz}), 2.01(3 \mathrm{H}, \mathrm{s})$, 2.76-2.92 $(1 \mathrm{H}, \mathrm{m}), 6.73(1 \mathrm{H}, \mathrm{s}), 7.11(1 \mathrm{H}, \mathrm{d}, \mathrm{J}=7.9 \mathrm{~Hz}), 7.21(1 \mathrm{H}, \mathrm{d}$, $\mathrm{J}=7.9 \mathrm{~Hz}), 7.25(1 \mathrm{H}, \mathrm{t}, \mathrm{J}=7.9 \mathrm{~Hz}), 7.75(1 \mathrm{H}, \mathrm{d}), 7.81(1 \mathrm{H}, \mathrm{t}, \mathrm{J}=7.9 \mathrm{~Hz})$, $7.91(1 \mathrm{H}, \mathrm{d}, \mathrm{J}=7.9 \mathrm{~Hz}),{ }^{13} \mathrm{C} \mathrm{NMR}\left(100 \mathrm{MHz}, \mathrm{CDCl}_{3}\right) \delta 15.94,23.15$, $33.41,77.88,116.43-116.65,128.11,131.35-132.19,148.14-148.21$, 167.29, FT-IR (KBr, cm $\left.{ }^{-1}\right) 3253$ and 2972 (Aromatic C-H Stretch), 1527 (Aromatic C=C Bending), 1423 (C-F Stretching), 1278 and $1201\left(-\mathrm{SO}_{2}\right.$ Stretching), 1024 (C-O Stretching), 721 (Aromatic C-H Bending), LCMS m/z calculated $\mathrm{C}_{16} \mathrm{H}_{17} \mathrm{BrO}_{3} \mathrm{~S}: 369.27$ found: $[\mathrm{M}+\mathrm{Na}]^{+} 393.40$.

5-isopropyl-2-methylphenyl 4-bromobenzenesulfonate (6f): ${ }^{1} \mathrm{H}$ NMR $\left(400 \mathrm{MHz}, \mathrm{CDCl}_{3}\right) \delta 1.12-1.14(6 \mathrm{H}, \mathrm{d}, \mathrm{J}=6.87 \mathrm{~Hz}), 2.01(3 \mathrm{H}, \mathrm{s})$, 2.76-2.92 $(1 \mathrm{H}, \mathrm{m}), 6.73(1 \mathrm{H}, \mathrm{s}), 7.02(1 \mathrm{H}, \mathrm{d}, \mathrm{J}=7.9 \mathrm{~Hz}), 7.21(1 \mathrm{H}, \mathrm{d}$, $\mathrm{J}=7.9 \mathrm{~Hz}), 7.46(2 \mathrm{H}, \mathrm{d}, \mathrm{J}=7.9 \mathrm{~Hz}), 7.91(2 \mathrm{H}, \mathrm{d}, \mathrm{J}=7.9 \mathrm{~Hz}),{ }^{13} \mathrm{C} \mathrm{NMR}$ $\left(100 \mathrm{MHz}, \mathrm{CDCl}_{3}\right) \delta 15.93,23.15,33.41,76.06-77.49,116.43-116.65$, 128.11, 131.35-132.19, 148.16-148.21, 164.14, FT-IR (KBr, cm $\left.{ }^{-1}\right) 2964$ (Aromatic C-H Stretch), 1562 (Aromatic C=C Bending), 1479 (C-F Stretching), 1377 and 1282 (-SO $\mathrm{SO}_{2}$ Stretching), 1083 (C-O Stretching), 806 (Aromatic C-H Bending), LC-MS m/z calculated $\mathrm{C}_{16} \mathrm{H}_{17} \mathrm{BrO}_{3} \mathrm{~S}$ : 369.27 found: $[\mathrm{M}+\mathrm{Na}]^{+} 393.40$.

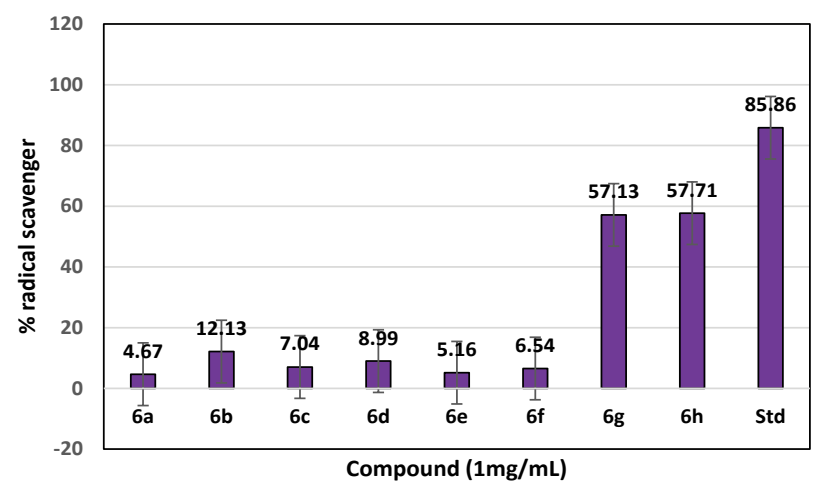

Figure 2: DPPH radical scavenging assay of synthesized compounds (6 a-h) and ascorbic acid. 


\begin{tabular}{|c|c|c|c|c|}
\hline Entry & Chemical Structure & M.P/B.P $P^{*}\left({ }^{\circ} \mathrm{C}\right)$ & Yield $^{\mathrm{b}}(\%)$ & (\%) Radical scavenger \\
\hline 1 & & $56-58^{*}$ & 91.13 & 4.67 \\
\hline 2 & & $86-88$ & 78.59 & 12.13 \\
\hline 3 & & $104-106$ & 85.45 & 7.04 \\
\hline 4 & & $98-100^{*}$ & 72.00 & 8.99 \\
\hline 5 & & $114-116$ & 83.26 & 5.16 \\
\hline 6 & & $64-68$ & 89.16 & 6.54 \\
\hline 7 & & $92-94$ & 82.95 & 57.13 \\
\hline 8 & & $76-78^{*}$ & 84.49 & 57.71 \\
\hline
\end{tabular}

aReaction conditions: Carvacrol (0.006 moles) and benzenesulfonylchlorides ( 0.006 moles), Triethylamine ( 0.009 moles); " blsolated Yield; 'BP Table. 1: Synthesis of benzenesulfonate derivatives using Carvacrol and benzenesulfonylchloridesa. 
5-isopropyl-4-methylphenylbenzenesulfonate $\quad(6 \mathrm{~g}):{ }^{1} \mathrm{H} \quad \mathrm{NMR}$ $\left(400 \mathrm{MHz}, \mathrm{CDCl}_{3}\right) \delta 1.11-1.12(6 \mathrm{H}, \mathrm{d}, \mathrm{J}=6.87 \mathrm{~Hz}), 2.14(3 \mathrm{H}, \mathrm{s}), 2.41$ $(3 \mathrm{H}, \mathrm{s}), 2.75-2.77(1 \mathrm{H}, \mathrm{m}), 6.67(1 \mathrm{H}, \mathrm{s}), 6.71(1 \mathrm{H}, \mathrm{d}, \mathrm{J}=7.9 \mathrm{~Hz}), 7.95$ $(1 \mathrm{H}, \mathrm{d}, \mathrm{J}=7.9 \mathrm{~Hz}), 7.25(2 \mathrm{H}, \mathrm{d}, \mathrm{J}=7.9 \mathrm{~Hz}), 7.75(2 \mathrm{H}, \mathrm{d}, \mathrm{J}=7.9 \mathrm{~Hz}),{ }^{13} \mathrm{C}$ NMR $\left(100 \mathrm{MHz}, \mathrm{CDCl}_{3}\right) \delta 15.45,15.99,21.71,23.74,24.05,33.41$, $33.70,76.82-77.46,113.09,118.46,120.19,120.06,125.22$, 128.56$128.74,129.78,130.77,131.35,133.13,145.37,148.04-148.30,153.97$, FT-IR $\left(\mathrm{KBr}, \mathrm{cm}^{-1}\right): 2964$ (Aromatic C-H Stretch), 1590 (Aromatic C=C Bending), 1436 (C-F Stretching), 1363 and 1288 (-SO Stretching), 1091 (C-O Stretching), 808 (Aromatic C-H Bending), LC-MS m/z calculated $\mathrm{C}_{17} \mathrm{H}_{20} \mathrm{O}_{3} \mathrm{~S}: 304.40$ found: $[\mathrm{M}+\mathrm{Na}]^{+} 327.28$.

5-isopropyl-2-methylphenyl 3(trifluoromethyl)benzenesulfonate (6h): ${ }^{1} \mathrm{H}$ NMR $\left(400 \mathrm{MHz}, \mathrm{CDCl}_{3}\right) \delta 1.16(6 \mathrm{H}, \mathrm{d}, \mathrm{J}=6.87 \mathrm{~Hz}), 2.09(3 \mathrm{H}, \mathrm{s})$, 2.72-2.81 (1H, m), $6.65(1 \mathrm{H}, \mathrm{s}), 6.71(1 \mathrm{H}, \mathrm{d}, \mathrm{J}=7.9 \mathrm{~Hz}), 7.03(1 \mathrm{H}, \mathrm{d}, \mathrm{J}=7.9$ $\mathrm{Hz}), 7.64(1 \mathrm{H}, \mathrm{d}, \mathrm{J}=7.9 \mathrm{~Hz}), 7.25(1 \mathrm{H}, \mathrm{d}, \mathrm{J}=7.9 \mathrm{~Hz}), 7.92(1 \mathrm{H}, \mathrm{d}, \mathrm{J}=7.9 \mathrm{~Hz})$, $8.12(1 \mathrm{H}, \mathrm{d}, \mathrm{J}=7.9 \mathrm{~Hz}),{ }^{13} \mathrm{C} \mathrm{NMR}\left(100 \mathrm{MHz}, \mathrm{CDCl}_{3}\right) \delta 8.63,15.52,23.67$, $36.40,46.45,76.63-77.46,117.97,119.25,125.66,128.52,129.08,129.40$, $130.20,130.81,130.88,131.69,132.29,145.02,148.05,148.18,148.45$, 154.32, FT-IR $\left(\mathrm{KBr}, \mathrm{cm}^{-1}\right) 3051$ and 2801 (Aromatic C-H Stretch), 1521 (Aromatic C=C Bending), 1379 (C-F Stretching), 1269 and 1199 (-SO Stretching), 1055 (C-O Stretching), 877 (Aromatic C-H Bending), LC-MS $\mathrm{m} / \mathrm{z}$ calculated $\mathrm{C}_{17} \mathrm{H}_{17} \mathrm{~F}_{3} \mathrm{O}_{3} \mathrm{~S}: 358.38$ found: $[\mathrm{M}+3]^{+} 361.25$.

\section{Results and Discussion}

In order to obtain carvacrol-phenylsulfonyl derivatives, carvacrol was treated with substituted phenylsulfonyl chlorides to get desired compounds as indicated in Scheme 1. Synthesized derivatives were obtained in good yields. Purification of these derivatives was carried out by simple recrystallization.

\section{In vitro antioxidant activity}

The antioxidant activity of the synthesized compounds was evaluated by 2,2-diphenyl-1-picrylhydrazyl (DPPH) free radical scavenging method [30,31]. The decreasing capacity of DPPH radicals was determined by a decline in their absorbance at $517 \mathrm{~nm}$ prompted by antioxidants. The inhibitory effects of the synthesized compounds 6a-h on DPPH radical are presented as the value of $\%$ inhibition (Figure 2). All the synthesized carvacrol derivatives were dissolved to prepare a stock solution of 1 $\mathrm{mg} / \mathrm{mL}$ using DMSO. Fifty microliter solutions of compounds were added to $1 \mathrm{~mL}$ of a $0.1 \mathrm{mM}$ solution of DPPH in methanol. After $2 \mathrm{~h}$, absorbance values were measured at $517 \mathrm{~nm}$. Ascorbic acid was used as a standard. The decrease in absorbance of DPPH radical was caused by antioxidants because of the reaction between antioxidant molecules and radical, which resulted in the scavenging of the DPPH radicals. DPPH radical scavenging activity of the synthesized compounds was distinguished to be good to moderate as compared with the standard ascorbic acid. The value of \% inhibition of the compounds $\mathbf{6 g}(57.13 \%)$ and $\mathbf{6 h}(57.71 \%)$ has been found to be greater as compared to standard ascorbic acid (85.86\%) at the same concentration. While it appears that compounds $\mathbf{6 a}, \mathbf{6 b}, \mathbf{6 c}, \mathbf{6 d}, \mathbf{6 e}$, and $\mathbf{6 f}$ are a poor scavenger of the DPPH radical $(4.67 \%, 12.13 \%, 7.04 \%, 8.99 \%, 5.16 \%$, and $6.54 \%)$ as compared to ascorbic acid (85.86).

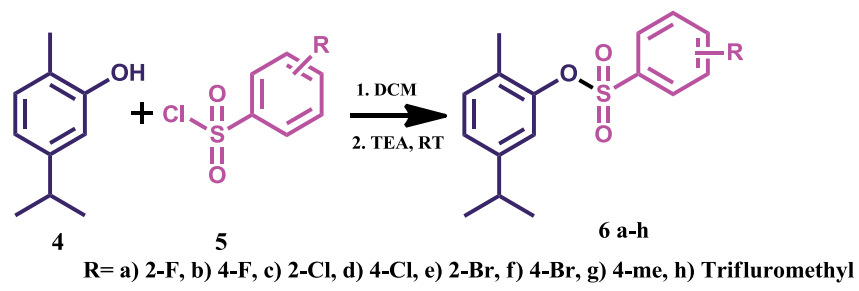

Scheme 1: Synthesis of Carvacrol-phenylsulfonyl derivatives.

\section{Conclusion}

We have synthesized eight new carvacrol benzenesulfonate derivatives $(\mathbf{6} \mathbf{a}-\mathbf{h})$ by simple and convenient methodology and characterized them by IR, ${ }^{1} \mathrm{H}$ and ${ }^{13} \mathrm{C}$ NMR and LC-MS spectroscopy. The comparison of the antioxidant activities of $\mathbf{6 a}-\mathbf{h}$ derivatives has revealed that the halo-substituted carvacrol benzenesulfonate derivatives have less antioxidant activity than the methyl and trifluoromethyl carvacrol benzenesulfonate derivatives. Amongst the series compounds, $\mathbf{6} \mathbf{g}$ and $\mathbf{6 h}$ exhibited remarkable antioxidant activity by DPPH radical scavenging assay (Table 1).

\section{Acknowledgements}

Ratnamala Bendre thanks to UGC-SAP (DSA-I), Suresh D. Bagul gratefully thanks to CSIR, New Delhi for financial assistance, and Jamatsing D. Rajput gratefully thanks, UGC, New Delhi for BSR Fellowship of PhD Candidates.

\section{References}

1. Kuş C, Ayhan-Kılcıgil G, Özbey S, Kaynak FB (2008) Synthesis and antioxidant properties of novel N-methyl-1, 3, 4-thiadiazol-2-amine and 4-methyl-2 $\mathrm{H}-1$, 2, 4-triazole-3 $(4 \mathrm{H})$-thione derivatives of benzimidazole class. Bioorg. Med. Chem. 16: 4294-4303.

2. Halliwell B, Gutteridge JM (2015) Free radicals in biology and medicine. Oxford University Press, USA.

3. Stief TW (2003) The physiology and pharmacology of singlet oxygen. Medical Hypotheses. 60: 567-572.

4. Tyagi YK, Kumar A, Raj HG, Vohra P, Gupta G, et al. (2005) Synthesis of nove amino and acetyl amino-4-methylcoumarins and evaluation of their antioxidant activity. Eur J Med Chem. 40: 413-420.

5. Karaaslan C, Kadri H, Coban T, Suzen S, et al. (2013) Synthesis and antioxidant properties of substituted 2-phenyl-1H-indoles. Bioorg. Med. Chem. Lett. 23: 2671-2674.

6. Maxwell SR (1995) Prospects for the use of antioxidant therapies. Drugs 49: 345-361.

7. Lucarini M, Mugnaini V, Pedulli GF (2002) Bond dissociation enthalpies of polyphenols: the importance of cooperative effects. The Journal of Organic Chemistry. 67: 928-931.

8. Snelgrove DW, Lusztyk J, Banks JT, Mulder P, Ingold KU, et al. (2001) Kinetic Solvent Effects on Hydrogen-Atom Abstractions: Reliable, Quantitative Predictions via a Single Empirical Equation. J. Am. Chem. Soc. 123: 469-477.

9. Baratta MT, Dorman HJ, Deans SG, Figueiredo AC, Barroso JG, et al. (1998) Antimicrobial and antioxidant properties of some commercial essential oils. Flavour and Fragrance Journal 13: 235-244.

10. Juven BJ, Kanner J, Schved F (1994) Factors that interact with the antibacterial action of thyme essential oil and its active constituents. J. Appl. Bacteriol. 76: 626-631.

11. Ruberto G, Baratta MT (2000) Antioxidant activity of selected essential oil components in two lipid model systems. Food Chem. 69: 167-174.

12. Chaieb K, Hajlaoui H, Zmantar T, Kahla-Nakbi AB, Rouabhia M, et al. (2007) The chemical composition and biological activity of clove essential oil Eugenia caryophyllata (Syzigium aromaticum L. Myrtaceae): a short review. Phytotherapy Research 21: 501-506.

13. Bagul SD, Rajput JD, Tadavi SK, Bendre RS (2017) Design, synthesis and biological activities of novel 5-isopropyl-2-methylphenolhydrazide-based sulfonamide derivatives. Research on Chemical Intermediates 43: 2241-2252.

14. Vale-Silva L, Silva MJ, Oliveira D, Gonçalves MJ, Cavaleiro C, et al. (2012) Correlation of the chemical composition of essential oils from Origanum vulgare subsp. virens with their in vitro activity against pathogenic yeasts and filamentous fungi. Journal of Medical Microbiology 61: 252-260.

15. Rajput JD, Bagul SD, Hosamani AA, Patil MM, Bendre RS, et al. (2017) Synthesis, characterizations, biological activities and docking studies of novel dihydroxy derivatives of natural phenolic monoterpenoids containing azomethine linkage. Research on Chemical Intermediates 43: 5377-5393.

16. Friedman $M$ (2014) Chemistry and multibeneficial bioactivities of carvacro (4-isopropyl-2-methylphenol), a component of essential oils produced by aromatic plants and spices. J. Agric. Food. Chem. 62: 7652-7670. 
Citation: Bagul SD, Rajput JD, Patil MM, Bendre RS (2017) Synthesis, Characterization and Antioxidant Activity of Carvacrol Based Sulfonates. Med Chem 7: 294-298. doi: 10.4172/2161-0444.1000470

17. Rajput JD, Bagul SD, Tadavi SK, Karandikar PS (2016) Design, synthesis and biological evaluation of novel class diindolyl methanes (DIMs) derived from naturally occurring phenolic monoterpenoids. Med. Chem. (Los Angeles) 6: 123-128.

18. Rajput JD, Bagul SD, Bendre RS (2017) Design, synthesis, biological screenings and docking simulations of novel carvacrol and thymol derivatives containing acetohydrazone linkage. Research on Chemical Intermediates 43: 4893-4906.

19. Damasceno SR, Oliveira FR, Carvalho NS, Brito CF (2014) Carvacryl acetate, a derivative of carvacrol, reduces nociceptive and inflammatory response in mice. Life Sciences. 94: 58-66.

20. Varel VH (2002) Carvacrol and thymol reduce swine waste odor and pathogens: stability of oils. Current Microbiology. 44: 38-43.

21. Suntres ZE, Coccimiglio J, Alipour M (2015) The bioactivity and toxicological actions of carvacrol. Crit. Rev. Food Science and Nutrition. 55: 304-318.

22. Jaberian H, Piri K, Nazari J (2013) Phytochemical composition and in vitro antimicrobial and antioxidant activities of some medicinal plants. Food Chem. 136: $237-244$.

23. Chaieb K, Hajlaoui H, Zmantar T, Kahla-Nakbi AB, Rouabhia M, et al. (2007) The chemical composition and biological activity of clove essential oil, Eugenia caryophyllata (Syzigium aromaticum L. Myrtaceae): a short review. Phytotherapy Research. 21: 501-506.
24. Pete UD, Zade CM, Bhosale JD, Tupe SG (2012) Hybrid molecules of carvacrol and benzoyl urea/thiourea with potential applications in agriculture and medicine. Bioorg. Med. Chem. Lett. 22: 5550-5554.

25. Friedman M (2013) Anticarcinogenic, cardioprotective, and other health benefits of tomato compounds lycopene, a-tomatine, and tomatidine in pure form and in fresh and processed tomatoes. J. Agric. Food. Chem. 61: 9534-9550.

26. Bayramoglu G, Senturk H, Bayramoglu A, Uyanoglu M, Colak S, et al. (2014) Carvacrol partially reverses symptoms of diabetes in STZ-induced diabetic rats. Cytotechnology. 66: 251-257.

27. Mastelic J, Jerkovic I, Blažević I, Poljak-Blaži M, Borovic S, et al. (2008) Comparative study on the antioxidant and biological activities of carvacrol, thymol, and eugenol derivatives. J. Agric. Food. Chem. 56: 3989-3996.

28. Yasuda D, Takahashi K, Kakinoki T, Tanaka Y, Ohe T, et al. (2013) Synthesis, radical scavenging activity and structure-activity relationship of uric acid analogs. Med Chem Comm. 4: 527-529.

29. Son S, Lewis BA (2002) Free radical scavenging and antioxidative activity of caffeic acid amide and ester analogues: structure activity relationship. J. Agric. Food. Chem. 50: 468-472.

30. Uppal G, Bala S, Kamboj S (2011) Therapeutic review exploring antimicrobial potential of hydrazones as promising lead. Der Pharma Chemica 3: 250-268.

31. Rane YS, Varma RR, Patil LS, Athlekar SV, Chowdhary AS, et al. (2010) Synthesis and antimicrobial activity of 5-substituted-2-(1-H-benzimidazole) sulfonamides. Asian Journal of Research in Chemistry (AJRC) 3: 335-338. 